\title{
Evaluation of Gall Bladder Volume in Type 2 Diabetes Mellitus Patients Using Real Time Ultrasonography
}

\author{
KS Jagadeesh ${ }^{\circledR 1}$, Ashwini M. Patil ${ }^{\odot 2}$ \\ ${ }^{1}$ Associate Professor, Department of Radiology, Akash Institute of Medical Sciences \& Research Centre, Devanahalli, Bengaluru, Karnataka, India , ${ }^{2}$ Assistant Professor, \\ Department of Radiology, Akash Institute of Medical Sciences \& Research Centre, Devanahalli, Bengaluru, Karnataka, India.
}

\section{Abstract}

Background: Diabetes mellitus is a group of metabolic diseases characterized by chronic hyperglycemia resulting from defects in insulin secretion, insulin action, or both. Autonomic neuropathy manifests as esophageal dysfunction, nocturnal diarrhea, gall bladder dysfunction, sphincter disturbances, atonic bladder and orthostatic hypotension. The present study aimed to evaluate and compare the gall bladder volume in fasting and post prandial state by real time ultrasound in Type 2 Diabetes Mellitus (T2DM) patients and healthy controls. Subjects and Methods: In this cross sectional study, 90 subjects were included. Among them, 45 were type 2 diabetes mellitus patients included as cases and 45 age and sex matched healthy controls, who attended the Department of Medicine and Radio-diagnosis, Akash Institute of Medical Sciences \& Research Centre, Devanahalli, Bengaluru, Karnaaka. All the study subjects were underwent detailed general and systemic examinations. Under aseptic conditions, $3 \mathrm{ml}$ fasting blood samples were collected and used for the estimation of fasting blood sugar, post prandial blood sugar. Gall bladder volume evaluation in fasting and 45 minutes post prandial (standardized fatty meal) state were done in T2DM patients and controls using real time ultrasound (GE Voluson P8 Mechine). Results: In the present study, BMI $\left(24.78 \pm 2.31 \mathrm{~kg} / \mathrm{m}^{2}\right), \mathrm{FBS}(160.98 \pm 27.99 \mathrm{mg} / \mathrm{dl}), \mathrm{PPBS}$ $(244.31 \pm 38.91 \mathrm{mg} / \mathrm{dl})$, Fasting gall bladder volume $\left(33.33 \pm 6.42 \mathrm{~cm}^{3}\right)$, post fatty meal gall bladder volume $\left(15.21 \pm 6.39 \mathrm{~cm}^{3}\right)$, ejection fraction $\left(49.34 \pm 17.29 \mathrm{~cm}^{3}\right)$ were significantly increased in type 2 diabetes mellitus patients compared with healthy controls. Conclusion: The study results conclude that, fasting and post-prandial gallbladder volumes are indicative of gallbladder function. Patients with type 2 diabetes mellitus showed statistically significant impairment of gallbladder function. Gallbladder function may be evaluated routinely in type 2 diabetes mellitus patients.

Keywords: Gallbladder volume, Diabetes mellitus, Ultrasonography

Corresponding Author: KS Jagadeesh, Associate Professor, Department of Radiology, Akash Institute of Medical Sciences \& Research Centre, Devanahalli, Bengaluru, Karnataka, India .

E-mail: jagadeesh_ks5@yahoo.co.in

Received: 02 February 2020

Revised: 01 March 2020

Accepted: 12 March 2020

Published: 05 July 2020

\section{Introduction}

Diabetes mellitus is a group of metabolic diseases characterized by chronic hyperglycemia resulting from defects in insulin secretion, insulin action, or both. Metabolic abnormalities in carbohydrates, lipids, and proteins result from the importance of insulin as an anabolic hormone. ${ }^{[1]}$ It is one of the chronic non-communicable diseases (CNCDs) which have emerged as a leading global health problem. It is also a known risk factor for blindness, vascular brain diseases, renal failure, and limb amputations. ${ }^{[2]}$

DM is a large problem worldwide. A total of 424.9 million adults have been estimated to have had DM, and this is estimated to rise to 628.6 million patients. The WHO eastern Mediterranean region has the highest prevalence of DM in the world. Type 2 diabetes mellitus (T2DM) is the major type of DM, accounting for approximately $90 \%$ of all cases. ${ }^{[3]}$ The risk factors of diabetes mellitus include urban residence, obesity, male sex, physical inactivity, increasing age, family history of diabetes and hypertension. ${ }^{[4-6]}$

Acute complications of diabetes mellitus include diabetic ketoacidosis, hyperglycemic state, hypoglycemia, and thrombosis and electrolyte disturbance. Therefore, premature morbidity, mortality, and the financial burden of diabetes result in a public health problem and are responsible for $10.7 \%$ global all-cause mortality among people aged between 20 to 70 years.

Autonomic neuropathy manifests as esophageal dysfunction, nocturnal diarrhea, constipation, gall bladder dysfunction, sphincter disturbances, atonic bladder, orthostatic hypotension, pilomotor disturbances and impotence. ${ }^{[7]} \mathrm{T} 2 \mathrm{DM}$ patients 
tend to have larger gall bladder volume than normal people, along with less vigorous contraction in response to fatty meals, predisposing these patients to cholelithiasis. ${ }^{[8]}$

Individuals with diabetes mellitus are reported to have a twofold to threefold increase in the incidence of cholesterol gallstones. A frequently cited but unproven pathophysiologic mechanism for this phenomenon is reduced gallbladder muscle function, which results in stasis and allows for cholesterol gallstone crystal formation and gallstone growth. To date, gallbladder motor function has not been investigated in a wellcharacterized diabetic population. ${ }^{[9]}$

Incomplete gall bladder emptying leads to sequestration of cholesterol and nidus formation. Therefore gall bladder functions should be evaluated routinely in such patients and early intervention is recommended. Acute complications of gall stones in diabetic patients are higher than in nondiabetic patients leading to suggestions of early elective cholecystectomy in diabetic patients with gall stones

A two to three fold increase in the risk of cholesterol gall stones has been reported in diabetic patients. ${ }^{[7]}$ Sonographic evaluation of gall bladder volume is a simple, non-invasive reproducible imaging parameter to evaluate volume and predict the cholesterol crystal and gall stone formation. Ultrasound is a reliable technique for evaluation of gall bladder volume. Emergency surgery in cases of acute complications of Cholelithiasis and operative mortality in diabetic patients are 4 to 5 times higher than in non-diabetic patients, leading to suggestions of early elective cholecystectomy in diabetic patients with cholelithiasis and also when evidence of nonfunctioning gall bladder is demonstrated. ${ }^{[10,11]}$

A noninvasive investigation like ultrasound for detection of pre and post prandial gall bladder volume in diabetic patients reveal the functional status of the gall bladder and can facilitate decision regarding early intervention. Gall bladder functions should be routinely evaluated in diabetic patients. The present study aimed to evaluate and compare the gall bladder volume in fasting and post prandial state by real time ultrasound in T2DM patients and healthy controls.

\section{Subjects and Methods}

In this cross sectional study, 90 subjects were included. Among them, 45 were type 2 diabetes mellitus patients with duration of 5 years or more, with diabetic complications are included as cases and 45 age and sex matched healthy controls, who attended the Department of Medicine and Radio-diagnosis, Akash Institute of Medical Sciences \& Research Centre, Devanahalli, Bengaluru, Karnaaka. A detailed medical history, presenting complaints, duration of diabetes mellitus, family history of diabetes mellitus, mode of treatment, exercise, diet, oral hypoglycemics, insulin, whether the treatment was regular, and history of diabetic complications were recorded. All the study subjects were underwent detailed general and systemic examinations. Peripheral neuropathy was assessed by presence of tingling and numbness in the palms and soles. Autonomic neuropathy was assessed by the presence of symptoms like dysphagia, abdominal fullness, nausea, vomiting, diarrhea, fecal incontinence, urinary incontinence, gustatory sweating, impotence etc. Under aseptic conditions, $3 \mathrm{ml}$ fasting blood samples were collected from all the subjects and used for the estimation of fasting blood sugar, post prandial blood sugar.

Gall bladder volume evaluation in fasting [Figure 1] and 45 minutes post prandial (standardized fatty meal) [Figure 2] state were done in type 2 diabetes mellitus patients and controls using real time ultrasound. Patients who have undergone previous cholecystectomy, acute or chronic hepatocellular disease and liver cirrhosis, patients with jaundice, gall bladder anomalies or diseases were excluded. Informed consent of the patients and controls were obtained and confidentially maintained.

\section{Ultrasound evaluation of gall bladder}

Gall bladder was evaluated by GE voluson P8.

\section{Positioning}

Patient was put in supine position with Radiologist sitting on the right side of the patient. Ultrasound probe positioned gently on the right hypochondriac region.

\section{Technique for visualization of Gall bladder}

\section{Supine position}

The gall bladder was first examined with the patient in a supine position. The long axis was obtained by varying the obliquity of the transducer until the maximum length of the gall bladder is seen. The maximum long axis $(\mathrm{H})$ was measured in centimeters.

Next the patient was scanned through the short axis of the gall bladder, beginning at the neck and sweeping through the fundus. A little angulation caudally through the body will demonstrate the entire fundus wall. In the region of body the maximum short axis dimension was selected and two cross sectional diameters, width (W) (Cranio caudal) and Anteroposterior diameter (AP) was measured in centimeters.

\section{Conventional ultrasound parameters measured}

Gall bladder volume was measured in fasting T2DM patients and in control subjects. Gallbladder volume was again measured in post prandial (standardized fatty meal) state in T2DM patients and controls.

Gall bladder volume was calculated using three measurements, these are.

Longitudinal axis $(\mathrm{H}$ 

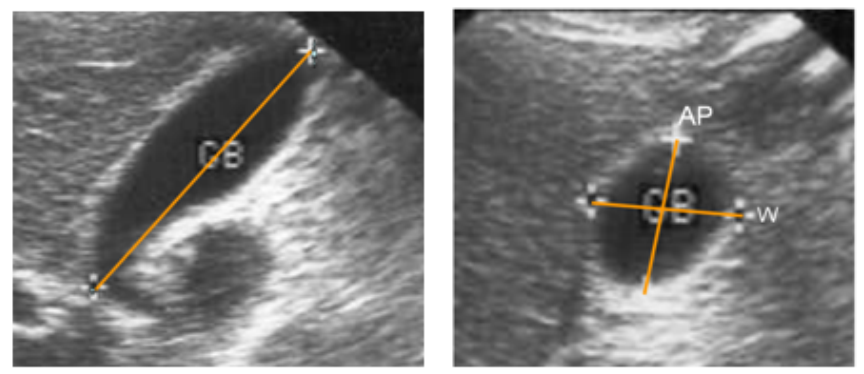

\section{Figure 1: Measurements of fasting Gall Bladder}
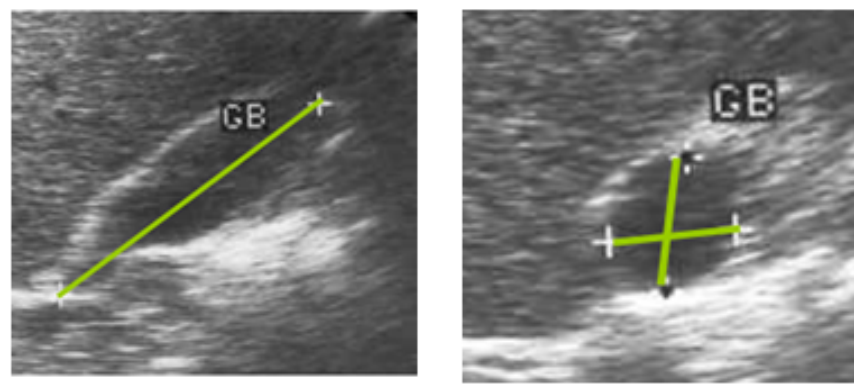

Figure 2: Measurements of Post - Fatty Meal Gall Bladder

Two short axis (Cross sectional diameters

Width (W

Antero-posterior diameter (AP

Using ellipse or ellipsoid formula the gall bladder volume was calculated

$\mathrm{V}=0.52(\mathrm{H} \times \mathrm{W} \times \mathrm{AP})$.

The percentage of gall bladder contraction (Ejection Fraction) was calculated by the formula

Ejection Fraction $(\mathrm{EF} \%)=$ Volume X1OO

Fasting GB Volume

The T2DM patients and the control subjects were given standardized fatty meal containing

Gm of fat containing $320+20 \mathrm{~K}$ calories

Two standard menus were chosen:

1.4 slice of bread $+40 \mathrm{gm}$ of butter ( 5 teaspoons of butter) + Jam.

Slices of bread +3 eggs (As omlette)

\section{Statistical analysis}

Independent student ' $t$ ' test was used to test the significance in both type 2 diabetes mellitus patients and control subjects. $\mathrm{p}<$
0.05 was considered as statistically significant. Data analysis was carried out using Statistical Package for Social Science (SPSS), Version 22.0

\section{Results}

In the present study, 45 type 2 diabetes mellitus patients as cases and 45 healthy subjects underwent sonographic evaluation of the gallbladder volume in both fasting state and post prandial (after standardized fatty meal) state. Laboratory parameters like fasting blood sugar and post prandial blood sugar were measured in all the subjects. In this study, mean age of the T2DM patients was $44.02 \pm 9.33$ and in the healthy controls $51.85 \pm 7.03(p=0.480)$. In the cases, 31 were males and 14 were females and in the control group 34 were males and 11 were females.

In the present study, BMI $\left(24.78 \pm 2.31 \mathrm{~kg} / \mathrm{m}^{2}\right)$, FBS $(160.98 \pm 27.99 \mathrm{mg} / \mathrm{dl})$, PPBS $(244.31 \pm 38.91 \mathrm{mg} / \mathrm{dl})$, Fasting gall bladder volume $\left(33.33 \pm 6.42 \mathrm{~cm}^{3}\right)$, post fatty meal gall bladder volume $\left(15.21 \pm 6.39 \mathrm{~cm}^{3}\right)$, ejection fraction $\left(49.34 \pm 17.29 \mathrm{~cm}^{3}\right)$ were significantly increased in type 2 diabetes mellitus patients compared with healthy controls as shown in [Table 1].

In the study group, T2DM patients were also subdivided broadly into without complications and those with diabetic complications. T2DM patients without diabetic complications were reported in $23(51.1 \%)$ patients. Peripheral neuropathy was observed in $9(20 \%)$, peripheral neuropathy \& Autonomic neuropathy was reported in $8(17.8 \%)$ cases as shown in [Table 2].

In the present study, fasting GB Volume $\left(\mathrm{cm}^{3}\right)$, post fatty meal GB Volume $\left(\mathrm{cm}^{3}\right)$ and ejection fraction (\%) were compared between the T2DM patients without complications and with complications. The fasting GB Volume $\left(\mathrm{cm}^{3}\right)$ was not statistically significant between two groups $(\mathrm{p}=0.275)$. The post fatty meal gallbladder volume $\left(\mathrm{cm}^{3}\right)$ was statistically significant between the two groups $(p<0.001)$. The ejection fraction (\%) difference observed in the mean value between the two groups was statistically significant with a $\mathrm{p}<0.02$ [Table 3 $\& 4]$. The mean duration was 10.76 years.

\section{Discussion}

Late complications of Diabetes mellitus are a major source of morbidity and mortality. Diabetics in particular those with T2DM have an increased prevalence of gallstones. ${ }^{[12]}$ Diabetic subjects are reported to have a two to three fold increase in the prevalence of cholesterol gall stones. ${ }^{[8]}$

Chapmann et al. conducted a large study involving 271 diabetic subjects, reported that there is increased incidence of cholesterol gallstones in T2DM patients. A highly significant 
Table 1: Comparison of BMI, FBS, PPBS, FGBV, PPGBV between T2DM \& Controls

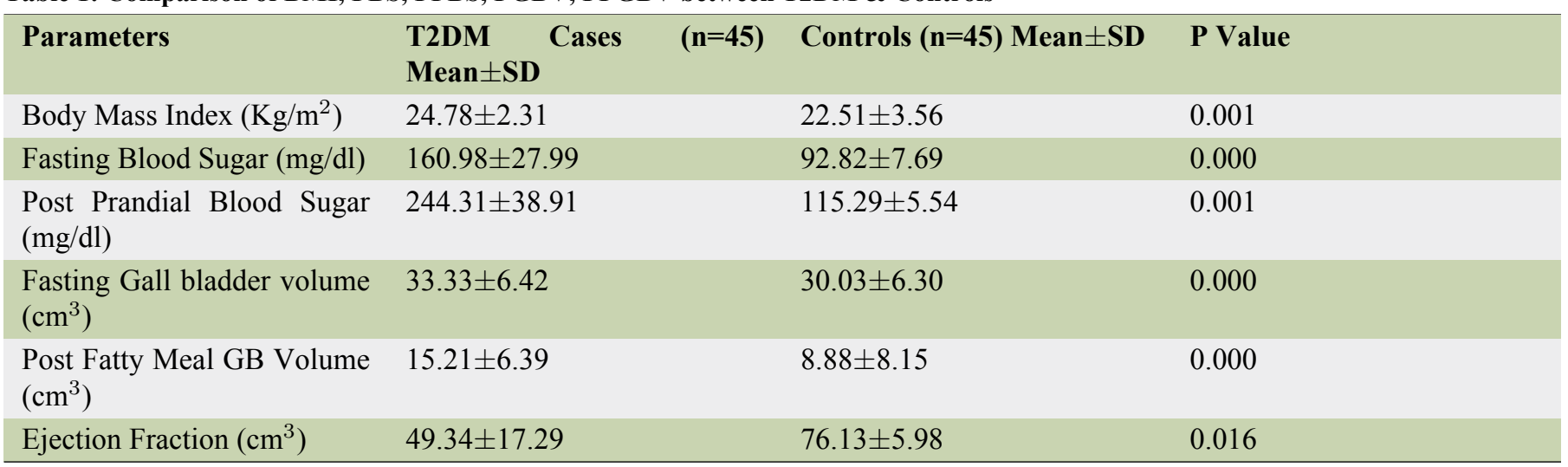

Table 2: T2DM patients without and with diabetic complications

\begin{tabular}{ll}
\hline & Number (\%) \\
Without Complications & $23(51.1 \%)$ \\
Peripheral Neuropathy & $9(20 \%)$ \\
Peripheral Neuropathy + Autonomic Neuropathy & $8(17.8 \%)$ \\
Peripheral Neuropathy + Retinopathy & $1(2.2 \%)$ \\
Peripheral Neuropathy + Diabetic Nephropathy & $2(4.4 \%)$ \\
Peripheral Neuropathy + Autonomic Neuropathy + IHD & $1(2.2 \%)$ \\
Peripheral Neuropathy + Diabetic Nephropathy +post renal transplant & $1(2.2 \%)$ \\
Total & $\mathbf{4 5 ( 1 0 0 \% )}$ \\
\hline
\end{tabular}

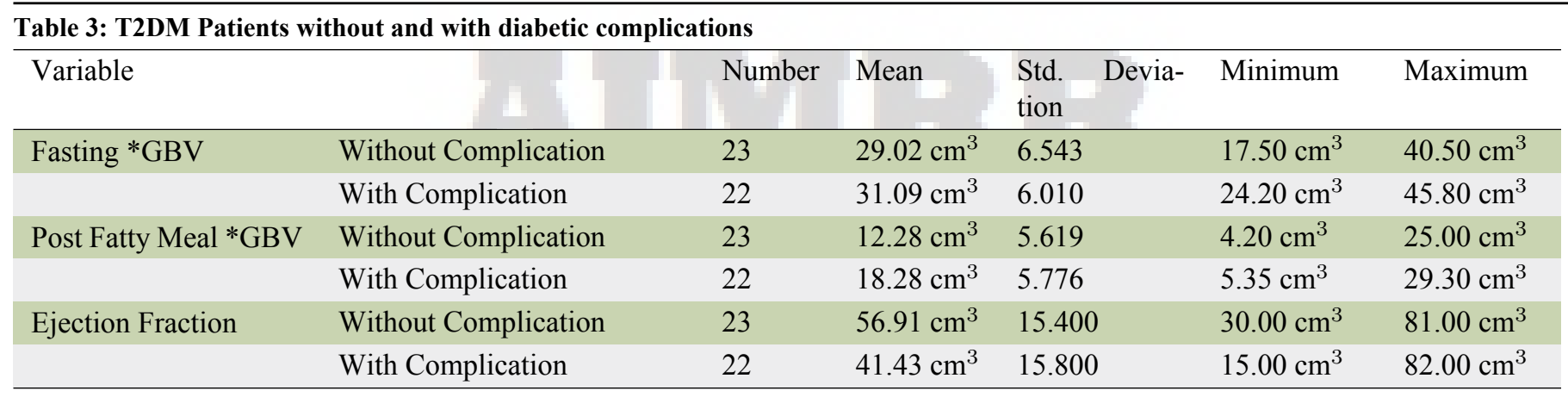

*GBV (Gall Bladder Volume)

Table 4: Independent Samples Test of T2DM patients without and with diabetic complications

\begin{tabular}{|c|c|c|c|}
\hline \multicolumn{4}{|c|}{ Independent Samples Test } \\
\hline & Student ' $t$ ' test & Df Degree of freedom & 'p' value \\
\hline Post Fatty Meal *GBV & -3.532 & 42.773 & .001 \\
\hline Ejection Fraction & 3.328 & 42.783 & .002 \\
\hline
\end{tabular}

*GBV (Gall Bladder Volume) 
increase in gall bladder volume was also observed in T2DM group. Conventional real time ultrasonography is a simple noninvasive investigation for evaluation of gall bladder volume. In a similar study C.GAUR et al who examined 40 patients of NIDDM, 10 patients of IDDM and 50 healthy controls, found that patients with NIDDM had statistically significant larger fasting gall bladder volume and these values were highly significant amongst patients with autonomic neuropathy. They also had significant larger post fatty meal gallbladder volume and these values were high in patients with autonomic neuropathy. ${ }^{\text {[7] }}$

In a study by PG Raman et al, ${ }^{[8]}$ who studied 50 NIDDM patients and 30 controls, found that $32 \%$ of diabetic patients had ultrasonographic evidence of gallstones as compared to $6.7 \%$ in healthy controls. They also found that mean fasting gall bladder volume was significantly increased in diabetic patients $\left(26 \mathrm{~cm}^{3}\right)$ as compared to control population $\left(15.8 \mathrm{~cm}^{3}\right)$. Furthermore, mean fasting gallbladder volume of diabetic patients with gallbladder disorders $\left(28.1 \mathrm{~cm}^{3}\right)$ was found to be significantly larger than that of those patients without gallbladder disorder $\left(24.6 \mathrm{~cm}^{3}\right)$

Mean percentage of contractions (Ejection fraction) of gallbladder 60 minutes after fatty meal was reduced in diabetic patients $(53 \%)$ and it was further reduced in the patients with gall bladder disorder (41.8\%). Mean duration of diabetes was significantly longer in diabetic patients with gallbladder disorder.

In a study by Agarwal AK et al., reported that mean fasting gallbladder volume in T2DM was $25.87 \pm 13.90 \mathrm{ml}$, with a minimum value of $9.30 \mathrm{ml}$ and maximum value of $88 \mathrm{ml}$ and higher gallbladder volumes were seen in patients with autonomic neuropathy. ${ }^{[13]}$

In a study by $\mathrm{C} A$ Ugbaja et al., reported that diabetic patients with neuropathy have significant

abnormalities of gall bladder function, presumably due to autonomic nerve dysfunction. These patients also have a higher prevalence of gallstones, suggesting that ultrasound screening may be useful. ${ }^{[14]}$

In a study conducted by Garjesh S. Rai et al., observed that higher fasting gall bladder volume and reduced percentage of contraction in T2DM patients attributed to autonomic neuropathy. Suggested that, hepatobiliary ultrasonography in chronic diabetics can be used as screening tool for early diagnosis of complication and to avoid its serious consequences when presents in emergency and undergone for surgery. ${ }^{[15]}$

In our study the fasting GB Volume $\left(\mathrm{cm}^{3}\right)$ difference was not statistically significant between T2DM patients without complications and T2DM patients with complications, but there was significant difference between the two in the post fatty gallbladder volume with a mean value of $12 \mathrm{~cm}^{3}$ in patients without complications and a mean value of $18.2 \mathrm{~cm}^{3}$ in patients with complications. Furthermore, percentage of ejection fraction showed significant difference with a mean value of $56.91 \%$ in patients without complications and a mean value of $41.43 \%$ in patients with complications.

There was significant difference in fasting gallbladder volume between the T2DM patients and the control group with a $p<0.016$. There was also significant difference in post fatty meal gallbladder volume between T2DM patients and control group with a $\mathrm{p}<0.001$. The difference in Ejection fraction (EF \%) between the two groups was also significant with a $\mathrm{P}<0.001$. Our study results matched the previous studies demonstrating the increased fasting GB Volume, post fatty meal GB Volume and decreased ejection fraction in the T2DM patients. ${ }^{[7,8]}$

Longer the mean duration of T2DM, there was an increase in the mean fasting GB Volume and decrease in ejection fraction (\%). In our study we also found there was a significant difference in Body Mass Index (BMI) between the T2DM patients and control group with a $\mathrm{p}<0.001$.

It is known that patients with diabetes often develop cholelithiasis and gall bladder stasis is considered to be one of the causes. ${ }^{[7]}$ Stasis leads to lithogenicity of bile and incomplete gall bladder emptying leading to sequestration of cholesterol and nidus formation, thereby predisposing to gallstone formation. The mechanism responsible for cholecystoparesis is attributed to vagal neuropathy.

\section{Conclusion}

Fasting and post-prandial gallbladder volumes are indicative of gallbladder function. Patients with T2DM show statistically significant impairment of gallbladder function as compared with normal controls. The impairment of gallbladder function is also related to complications and the duration of T2DM. Ultrasound evaluation of gall bladder volume (fasting and post-prandial) and Ejection Fraction are efficient parameters to evaluate gallbladder function. Gallbladder function should be evaluated routinely in T2DM patients as incomplete gallbladder emptying may lead to gallstone formation and associated complications. Further studies with large sample size are recommended.

\section{References}

1. Kharroubi AT. Diabetes mellitus: The epidemic of the century. World J Diabetes. 2015;6(6):850-854. Available from: https: //dx.doi.org/10.4239/wjd.v6.i6.850.

2. Aynalem SB, Zeleke AJ. Prevalence of Diabetes Mellitus and Its Risk Factors among Individuals Aged 15 Years and Above in Mizan-Aman Town. Int J Endocrinol. 2018;2018:9317987. Available from: https://doi.org/10.1155/2018/9317987. 
3. Omar SM, Musa IR, ElSouli A, Adam I. Prevalence, risk factors, and glycaemic control of type 2 diabetes mellitus in eastern Sudan: a community-based study. Ther Adv Endocrinol. 2019;10:1-8. Available from: https://dx.doi.org/ 10.1177/2042018819860071.

4. Wang S, Ma W, Yuan Z, Wang SM, Yi X, Jia H. Association between obesity indices and type 2 diabetes mellitus among middle-aged and elderly people in Jinan, China: a crosssectional study. BMJ Open. 2016;6(11):12742-12746. Available from: https://doi.org/10.1136/bmjopen-2016-012742.

5. Nayak SB, Rahming V, Raghunanan Y, Raghoonath C, Rahman D, Rajh. Prevalence of diabetes, obesity and dyslipidaemia in persons within high and low income groups living in North and South Trinidad. J Clin Diagn Res. 2016;10(5):8-13. Available from: https://dx.doi.org/10.7860/ JCDR/2016/18154.7875.

6. Babu GR, Murthy GVS, Ana Y, Patel P, R D, Neelon SEB, et al. Association of obesity with hypertension and type 2 diabetes mellitus in India: A meta-analysis of observational studies. World J Diabetes. 2018;9(1):40-52. Available from: https://dx.doi.org/10.4239/wjd.v9.i1.40.

7. Gaur C, Mathur, Agarwal A, Verma K, Jain R, Swaroop A. Diabetic autonomic neuropathy causing gallbladder dysfunction. J Assoc Phys India. 2000;48:603-605.

8. Raman PG, Patel A, V M. Gall bladder disorder and Type 2 diabetes mellitus. A clinical based study. J Assoc Phys India. 2002;50:887-890.

9. Stone BG, Gavaler JS, Belle SH, Shreiner DP, Peleman RR, Sarva RP, et al. Impairment of gallbladder emptying in diabetes mellitus. Gastroenterol. 1988;95(1):170-176. Available from: https://dx.doi.org/10.1016/0016-5085(88)90307-1.

10. Fraquelli M, Pagliarulo M, Colucci A, Paggi S, Conte D. Gallbladder motility in obesity, diabetes mellitus and coeliac disease. Digest Liver Dis. 2003;35:12-16. Available from: https://dx.doi.org/10.1016/s1590-8658(03)00087-2.

11. Steven M, Haffner, Andrew K. Clinical gallbladder disease in NIDDM subjects. Diabetes care. 1993;16(9):1276-1282. Available from: https://doi.org/10.2337/diacare.16.9.1276.

12. Chapman TMBA, Chapman CM, Frampton RJ, Chisholm RB, Allan IR, Wilson. Gallbladder volume comparison of diabetics and controls. Digestive Dis Sci. 1998;43(2):344-348. Available from: https://doi.org/10.1023/a:1018810523399.

13. Agarwal AK, Miglani S, Singla S, Garg U, Dudeja RK, Goel A. Ultrasonographic evaluation of gallbladder volume in diabetics. J Assoc Physicians India. 2004;52:962-967.

14. Ugbaja CA, Ayoola RTOO, Ikem BM, Idowu. Gall bladder volume and contractility in type 2 diabetes mellitus. Afr J Diabetes Med. 2015;23(2):9-12.

15. Rai G, Baghel V, Rai T, Vyas M. Gall bladder dysfunction in chronic diabetics (type 2): an ultrasonography based prospective study. Int J Res Med Sci. 2016;4(2):390397. Available from: https://dx.doi.org/10.18203/2320-6012. ijrms20160084.

Copyright: (C) the author(s), 2020. It is an open-access article distributed under the terms of the Creative Commons Attribution License (CC BY 4.0), which permits authors to retain ownership of the copyright for their content, and allow anyone to download, reuse, reprint, modify, distribute and/or copy the content as long as the original authors and source are cited.

How to cite this article: Jagadeesh KS, Patil AM. Evaluation of Gall Bladder Volume in Type 2 Diabetes Mellitus Patients Using Real Time Ultrasonography. Asian J. Med. Radiol. Res. 2020; 8(1):83-88.

DOI: dx.doi.org/10.47009/ajmrr.2020.8.1.15

Source of Support: Nil, Conflict of Interest: None declared. 